\title{
REVIEWS
}

\section{Proposal for the assessment of quantitative dermal exposure limits in occupational environments: part 1. Development of a concept to derive a quantitative dermal occupational exposure limit}

\author{
P M J Bos, D H Brouwer, H Stevenson, P J Boogaard, W L A M de Kort, J J van Hemmen
}

Abstract

Dermal uptake of chemicals at the workplace may contribute considerably to the total internal exposure and so needs to be regulated. At present only qualitative warning signs-the "skin notations"-are available as instruments. An attempt was made to develop a quantitative dermal occupational exposure limit (DOEL) complementary to respiratory occupational exposure limits (OELs). The DOEL refers to the total dose deposited on the skin during a working shift. Based on available data and experience a theoretical procedure for the assessment of a DOEL was developed. A DOEL was derived for cyclophosphamide and 4,4methylene dianiline (MDA) according to this procedure. The DOEL for MDA was tested for applicability in an actual occupational exposure scenario. An integrated approach is recommended for situations in which both dermal and respiratory exposures contribute considerably to the internal exposure of the worker. The starting point should be an internal health based occupational exposure limit-that is, the maximum dose to be absorbed without leading to adverse systemic effects. The proposed assessment of an external DOEL is then either based on absorption rate or absorption percentage. The estimation of skin penetration seems to be of crucial importance in this concept. If for a specific substance a maximal absorption rate can be estimated a maximal skin surface area to be exposed can be assessed which may then serve the purpose of a DOEL. As long as the actual skin surface exposed is smaller than this maximal skin surface area the internal OEL will not be exceeded, and therefore, no systemic health problems would be expected, independent of the dermal dosel unit area. If not, the DOEL may be interpreted as the product of dermal dose/ unit area $\left(\mathrm{mg} / \mathrm{cm}^{2}\right)$ and exposed skin surface area $\left(\mathrm{cm}^{2}\right)$. The proposed concept for a DOEL is relevant and can be made applicable for health surveillance in the occupational situation where dermal exposure contributes notably to the systemic exposure. Further research should show whether this concept is more generally applicable.

(Occup Environ Med 1998;55:795-804)

Keywords: dermal exposure; occupational exposure limits; industrial hygiene

BACKGROUND FOR DERMAL OCCUPATIONAL EXPOSURE LIMITS

At the workplace toxic substances may enter the body through the respiratory tract, through the gastrointestinal tract, and through the skin. It is assumed that the major routes of entry are the airways and the skin, although the intestinal route may not be negligible due to the so called hand-mouth shunt and, secondary ingestion after respiratory exposure.

To protect people form detrimental effects due to exposure to chemicals, several limit values have been developed. For oral exposure acceptable daily intake values (ADIs) have been adopted for the general population, but these are of limited value to workers. Several types of quantitative occupational exposure limits (OELs) have been derived to protect workers from adverse health effects of toxic substances at work. Basically, if exposure is kept below the limit, no adverse health events are expected in the workers (table 1). For respiratory exposure, the threshold limit value (TLV, set by the American Conference of Governmental Industrial Hygienists (ACGIH)) is most often adopted worldwide. In the Netherlands, maximum accepted concentrations (MAC values) are in use.

As well as external exposure limit values, internal values exist for several xenobiotics for systemic exposure, known as biological limit values (BLVs). Examples are the biological exposure index (set by the ACGIH) ${ }^{1}$ and the German "BAT-Werte" (set by the "Deutsche Forschungsgemeinschaft"). ${ }^{2}$ Recently, the Health and Safety Executive in the United Kingdom has introduced biological monitoring guidance values for six substances. ${ }^{3}$ The main advantages of a BLV relate to $(a)$ its independence of the route of entry, and $(b)$ its use in assessing an overall health risk, as monitoring of 
Table 1 Some characteristics of available exposure limits

\begin{tabular}{|c|c|c|c|c|}
\hline & \multicolumn{4}{|l|}{ Route of entry } \\
\hline & Respiratory tract & Gastrointestinal tract & Skin & Miscellaneous or combined \\
\hline Name & $\begin{array}{l}\text { Maximum accepted } \\
\text { concentration (MAC) } \\
\text { Threshold limit value (TLV) }\end{array}$ & Acceptable daily intake (ADI) & Skin denotation & $\begin{array}{l}\text { Biological limit value; (BEI, BAT-Werte, } \\
\text { biological monitoring guidance value) }\end{array}$ \\
\hline $\begin{array}{l}\text { Qualitative or } \\
\text { quantitative }\end{array}$ & Quantitative & Quantitative & Qualitative & Quantitative \\
\hline $\begin{array}{l}\text { Target population } \\
\text { Dimensions }\end{array}$ & $\begin{array}{l}\text { Working population } \\
\mathrm{mg} / \mathrm{m}^{3} \\
\text { parts per million }(\mathrm{ppm}) \\
\text { fibres } \mathrm{n} / \mathrm{m}^{3}\end{array}$ & $\begin{array}{l}\text { General population } \\
\mathrm{mg} / \mathrm{kg} \text { food } \\
\mathrm{mg} / \mathrm{kg} \text { body weight }\end{array}$ & $\begin{array}{l}\text { Working population } \\
\text { Not applicable; however } \\
\text { likely to be assessed as } \mathrm{mg} \\
\left(\mathrm{mg} / \mathrm{cm}^{2}\right)\end{array}$ & $\begin{array}{l}\text { Working population or general population } \\
\text { (a) } \mathrm{mg} / \mathrm{l} \text { blood, } \mathrm{mg} / \mathrm{l} \text { urine, } \mathrm{mg} / \mathrm{m}^{3} \text { exhaled air } \\
\text { (b) cholinesterase inhibition, zinc } \\
\text { protoporphyrin, DNA adducts, mutations, etc }\end{array}$ \\
\hline \multirow[t]{2}{*}{ Monitoring methods } & $\begin{array}{l}\text { Environmental monitoring } \\
(\mathrm{EM})\end{array}$ & $\begin{array}{l}\text { Food residues or } \\
\text { contaminants in combination } \\
\text { with food intake data }\end{array}$ & \multirow{2}{*}{$\begin{array}{l}\text { For example, } \\
\text { environmental surface } \\
\text { wipe-off; patches, gloves, } \\
\text { coveralls; tracer methods; } \\
\text { skin washings; or skin } \\
\text { stripping }\end{array}$} & \multirow[t]{2}{*}{$\begin{array}{l}\text { Biological media: blood, urine, exhaled air, } \\
\text { faeces, hair }\end{array}$} \\
\hline & Personal air sampling (PAS) & $\begin{array}{l}\text { No specific worker } \\
\text { monitoring method }\end{array}$ & & \\
\hline
\end{tabular}

internal (systemic) exposure provides information on the result of exposure through all possible routes of entry. Disadvantages are that local effects are not covered and only a few adequate monitoring methods based on human toxicokinetic data are available. Specific strategies for the sampling of biological fluids may be necessary. Furthermore, the position of blood sampling relative to the skin exposure may be critical for the outcome, ${ }^{4}$ and possible skin metabolism is not generally taken into consideration. $^{5}$

No quantitative limit values exist that may protect against adverse effects from uptake through dermal exposure. A qualitative "skin notation" is generally the only available warning against absorption of chemical substances through the skin. This, however, only points to a potential risk but gives no indication of the actual contribution of skin exposure to the body burden. For example, 2-methoxyethanol has a skin notation, but it was estimated by Paustenbach that dermal uptake by exposure of one hand for 30 minutes was 4.5 times the amount taken up by inhalation for eight hours to the TLV of 5 ppm. ${ }^{6}$ Furthermore, clear discrepancies in the denotation practice exist between countries. Besides, the absence of a skin notation does not imply that risk due to skin exposure can be ignored. ${ }^{7}$ The scientific basis underlying the skin notation in the TLV list of the ACGIH is not always well documented. ${ }^{8}$ In The Netherlands, a skin notation is applied by the Dutch Expert Committee on Occupational Standards (DECOS) predominantly based on a strategy proposed by European Centre for Ecotoxicology and Toxicology of Chemicals (ECETOC)..$^{9}$ It is well known that uptake through the skin of several chemicals can contribute considerably to the internal exposure. For many chemicals-for example, pesticides - the skin is the predominant route of entry. For adequate protection of workers against adverse health effects of these compounds, it is important to regulate exposure through dermal uptake rather than, or as well as, pulmonary uptake, with preference for a quantitative variable. ${ }^{10}$ The importance of regulating dermal exposure has also recently been stressed at a European meeting funded by the European Community, ${ }^{11}$ which led to the institution of the Dermal Exposure Network.
At the request of the Dutch Ministry of Social Affairs and Employment a concept was developed for a more quantitative health based dermal occupational exposure limit (DOEL). A practically applicable DOEL may serve as a useful tool in the policy of protecting workers' health and provide the government with a valuable and applicable instrument for the regulation of skin exposure at the workplace as well as other OELs. The process was divided into three phases, (a) the development of such a concept, $(b)$ assessment of a DOEL for two substances according to this concept, and (c) testing the applicability of one of these DOELs in an actual occupational environment. The present report describes the first two phases. The third phase is presented in an accompanying paper. ${ }^{12}$ The present reports are considered to serve as a starting point for the development of DOELs, and are not meant to be the final guidance for the derivation for a DOEL.

General aspects of setting priorities of substances and the different levels at which a DOEL may be set (internal or external exposure) are considered, and the toxicological basis for this is discussed. As a DOEL is meant to be an instrument to keep the internal dose below a certain level the estimation of the actual dose taken up will be crucial in this concept. This can be approached in two ways, absorption rate and absorption percentage. Both possibilities are presented followed by a discussion about the difficulties associated with the estimation of dermal uptake in the occupational situation. The uptake is dependent on many factors that will vary in different occupational settings. As illustrative examples, DOELs are calculated according to the proposed concept for cyclophosphamide and 4,4'methylene dianiline (MDA).

PRIORITY SETTING FOR THE ASSESSMENT OF DERMAL OCCUPATIONAL EXPOSURE LIMITS A DOEL, in combination with other types of standards, may provide a more complete set of exposure limits, which together allow for a risk assessment of a workers' health relative to chemical exposure at work (fig 1 ). It will be neither possible nor necessary to assess a DOEL for every possible substance. Assessment of a DOEL will be useful for all chemicals for which adequate protection of the worker demands 


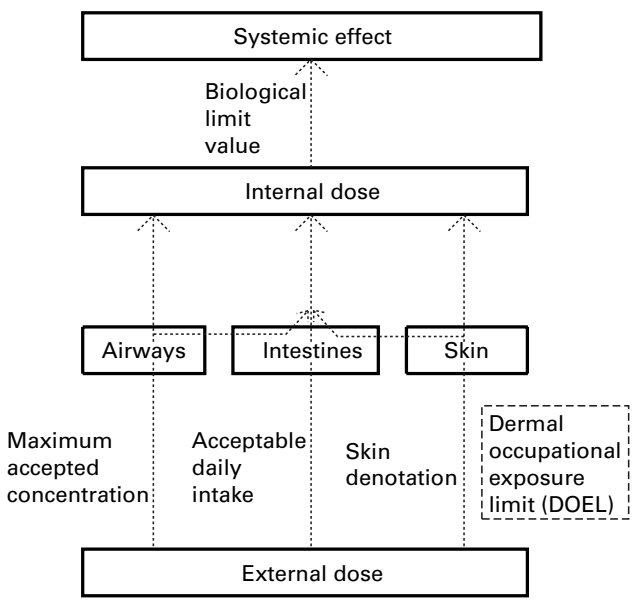

Figure 1 Exposure routes and limit values. For the dermal route the limit (skin denotation) is of a qualitative nature. The dermal occupational exposure limit (DOEL) could fill this gap.

monitoring skin exposure on a regular basis. Therefore, criteria should be developed to set a priority list of chemicals. Priority setting may be based on data obtained from different viewpoints and sources. Chemicals for which a skin notation could be assessed as well as an OEL for respiratory exposure may be selected. For example, Fiserova-Bergerova et al proposed the comparison of rates of skin penetration and pulmonary uptake at the level of an OEL as a criterion for relevant dermal uptake. ${ }^{13}{ }^{14}$ However, these chemicals are generally selected according to criteria for priority setting for respiratory exposure- for example, based on the prevention of respiratory tract irritation-which are not necessarily applicable to dermal exposure.

The ECETOC and the US Environmental Protection Agency (US EPA) published documents that may provide some useful information on this topic..$^{15}$ Further, specific groups of substances related to either structure or effect may be identified for which a DOEL is either necessary or unnecessary. Examples of substances related to structure are the glycol ethers, ${ }^{16}{ }^{17}$ whereas examples of those related to effect are defatting agents which may either be taken up quickly themselves, or increase skin uptake of other compounds present.

For physicochemical variables, a molecular weight $<500$ or a $\log P_{o w}$ between -1 and 4 may indicate potentially considerable dermal uptake; vapour pressure and boiling point are also important variables. ${ }^{15}$ 18-20 For toxicodynamic variables, dermal toxicity data compared with those obtained by other routes may provide suitable information - for example, comparison of a dermal $\mathrm{LD}_{50}$ and intravenous $\mathrm{LD}_{50}$ may be indicative, except in those cases where the rate of appearance in the systemic circulation is important. Also, actual or estimated occupational exposure conditions will also provide important information for priority setting. Models for the estimation of dermal exposure for specific activities have been developed; description of these models are beyond the scope of this report.

Occupational case reports of systemic toxic effects in the absence of respiratory exposurefor example, $\mathrm{MDA}^{12}$ - or urinary excretion patterns indicative of skin uptakecyclophosphamide $^{21}$ - may clearly point to the necessity of controlling dermal exposure rather than or as well as respiratory exposure.

\section{Basic elements for a dermal occupational} exposure limit

OUTLINE AND DEFINITIONS

The OELs are assessed to control the internal exposure of the worker exposed to chemicals, to serve to keep the internal exposure below a concentration or dose at which no adverse health effects are expected, or below an accepted level. Similarly, a DOEL should represent the maximum amount of substance (mg) deposited on the skin surface within a given time (usually a workshift), without giving rise to adverse systemic health effects.

Fenske defined dermal exposure as the product of skin loading rate (mass per skin surface area per unit time) and area exposed $\left(\mathrm{cm}^{2}\right){ }^{22}$ Dermal exposure is expressed in units of mass per unit time $(\mu \mathrm{g} / \mathrm{h})$. Cherrie and Robertson proposed an alternative definition which recognises the biological process involved in skin absorption - that is, the concentration of the substance at the skin surface. ${ }^{23}$

Dermal exposure at the workplace is generally intermittent. Due to the reservoir function of the skin, ${ }^{24}$ internal exposure may continue even after the external exposure has ended. Occupational exposure may be due to contact with contaminated surfaces, gases, aerosols, liquids, and dusts. Two expressions of dermal exposure have been defined..$^{25}$ Firstly, potential dermal exposure, which is the total amount of chemical (mg) deposited on the worker, either on the (protective) clothing or the bare (uncovered) skin. Secondly, actual dermal exposure, which is defined as the amount of a chemical actually coming into contact with the bare (uncovered) skin, including the fraction transferring through (protective) clothing to the underlying skin, and which is, therefore, available for percutaneous absorption. Preferably, insight on actual exposure conditions (frequency and duration of exposure, skin surface area, and location) should be present for the derivation of an accurate DOEL. Otherwise, a DOEL may turn out to be too conservative because of several conservative assumptions that have to be made.

POSSIBLE LEVELS FOR SETTING A DERMAL OCCUPATIONAL EXPOSURE LIMIT

It is obvious that a DOEL has to be assessed so that testing for compliance can be performed with (relative) ease. Theoretically, DOELs may be set at the following levels:

- The internal level (setting a BLV)

- The level on the skin surface (mg deposited on the skin)

- The level in the occupational environment (the amount of chemical present on surfaces of working equipment, or pesticide residues).

As already mentioned, the first level, assessment of a BLV, has clear advantages and is the level to be considered first. However, it is at the moment, limited in its use. The third level has 
been used to investigate crop harvesting activities where skin contact may occur with pesticide residues on the crop. ${ }^{26}$ In other occupational environments, it was thought that it would be more complex to set a DOEL at this level and to test it for compliance. Furthermore, regulating skin exposure at the third level is considered to be a derivation of the second level. Therefore, this report focuses on the second level, whether an applicable and useful external DOEL can be assessed for the amount of chemical deposited on the skin. It is hoped that the DOEL will be a useful tool in controlling the internal exposure as a result of skin uptake and should therefore be related to either the maximal internal dose that is expected not to affect the health of the worker, or to a generally accepted risk level.

BASIS FOR A DERMAL OCCUPATIONAL EXPOSURE LIMIT

The maximal internal dose (based on the no adverse observed effect level (NOAEL)) can either be derived from human data (preferably) or from animal data. Such data should relate the external dermal dose directly to health effects, leading automatically to an acceptable value of a DOEL. Suitable human data are usually lacking, therefore, data for other exposure routes or animal data may be used to derive a DOEL. Acceptance of a pivotal role for the internal exposure (concentration of the toxicant in the central compartment) is then essential in the process of the extrapolations from animal to human and route to route.

If based on animal data, data from dermal toxicity experiments are preferred, provided that experimental exposure conditions resemble those at the workplace (exposed surface area, dose or concentration per surface area, exposure time, climatological conditions, etc). ${ }^{27}$ The assessment of a DOEL from these experiments through direct extrapolation will generally include high to low dose extrapolation. It should then be taken into account that the absorption percentage may increase with a decreasing dermal area dose $\left(D_{A} \mathrm{mg} / \mathrm{cm}^{2}\right)$. In the case of a high $D_{A}$ (infinite dose ${ }^{18}$ ) a considerable amount of the substance may not be absorbed during the exposure period or workshift and may be finally wiped off; the absorption percentage will then be much less than $100 \%$ of the applied dose. However, for a low $D_{A}$ (finite dose ${ }^{18}$ ) the exposure period may be long enough for the deposited amount of substance to be (almost) completely (or maximally) absorbed. Thus an absorption percentage experimentally derived for a specific dermal dose/unit area cannot be generally applied to other doses. ${ }^{15} 18$ Linear extrapolation of an estimated absorption percentage to lower dermal doses may, therefore, result in an underestimation of the amount absorbed. It was concluded by ECETOC that absorption data expressed as a percentage of applied dose absorbed per unit of time are relevant only to a particular dose and a particular time. ${ }^{18}$

In the practice of setting standards, OELs for systemic effects have often to be based on oral or respiratory animal experiments, as suitable dermal toxicity studies are usually absent for most chemicals. In either case, route to route extrapo- lation has to be performed to derive an external DOEL. Based on these experiments an internal health based recommended OEL (HBR-OEL ${ }_{\text {int }}$ ) for the worker will then be derived and will serve as a basis for a DOEL. (A basis for this derivation is given in a recent report. ${ }^{28}$ ) The $\mathrm{HBR}^{-O E L_{\text {int }}}$ is defined as the maximal internal dose (mg/day) not leading to adverse health effects for the worker. A NOAEL is translated into an HBR-OEL ${ }_{\text {int }}$ with toxicokinetic data (mainly bioavailability data) for the exposure route the NOAEL is based on. An external DOEL is derived from an $\mathrm{HBR}_{-} \mathrm{OEL}_{\text {int }}$, with toxicokinetic data for dermal exposure-for example, dermal permeability constant, penetration flux, or absorption percentage. Because route to route extrapolation is the method extended farthest and most often applied in risk assessment for dermal exposure in the occupational situation, the proposed concept is based on this method. The final procedure proposed will also be applicable for direct extrapolation.

\section{Proposal for a standard: the dermal occupational exposure limit}

Crucial for the translation of an $\mathrm{HBR}-\mathrm{OEL}_{\text {int }}$ into an external DOEL as defined in the previous section, is the estimation of the dermal uptake which may be based on estimated or measured absorption rate or absorption percentage.

ASSESSMENT OF A DOEL BASED ON ABSORPTION RATE (FLUX)

If adequate data on absorption rate are available, a refined derivation of the DOEL is possible. For the undamaged skin, the variable for the steady state absorption rate is the flux $\mathcal{F}$ (mg/( $\mathrm{cm}^{2} \times$ hour)), defined as $K \times \Delta C$ (Fick's first law of diffusion), where $K$ is the permeability constant, and $\Delta C$ is the concentration gradient across the stratum corneum. ${ }^{15}$

The absorption rate is, among others, dependent on $D_{A}$ (actually on the concentration in the vehiculum), ${ }^{15}$ and will increase with increasing $D_{A}$ until a steady state flux is reached. A further increase of $D_{A}$ will then not result in a higher rate of uptake. For the purpose of the assessment of a DOEL, the maximal flux derived under exposure conditions relevant for the occupational situation $\left(\mathcal{F}_{\text {max;occ }}\right)$ should be the basis. The internal dose (mg) is then determined by the product $\mathscr{f}_{\text {max } ; 0 c} \times T \times A$, where $T$ is duration of exposure (hours/day) - that is, the time from the onset of dermal exposure until the exposure is ended and the skin is cleaned, and $A$ is the exposed skin surface area $\left(\mathrm{cm}^{2}\right)$. The maximal internal dose is then equal to $\mathcal{F}_{\text {max; } ; 0 c} \times T \times A$. Starting from a maximal accepted internal dose (HBR$\left.\mathrm{OEL}_{\text {int }}(\mathrm{mg} / \mathrm{day})\right)$, it follows:

$$
\begin{aligned}
& \mathcal{F}_{\text {max;occ }} \times T \times A \leqslant \text { HBR-OEL } \\
& \text { or } \\
& A \leqslant \mathrm{HBR}-\mathrm{OEL}_{\text {int }} /\left(\mathcal{F}_{\text {max; ;occ }} \times T\right) .
\end{aligned}
$$

So, under the assumption of a specified exposure time (default: $T=8$ hours/day) the internal dose depends only on the exposed skin surface area $A$. This means that a maximal allowable exposed skin surface area $\left(A_{\max }\right)$ can 


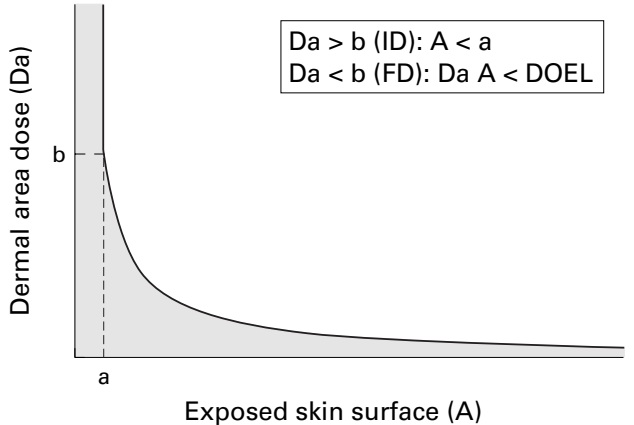

Figure 2 Graphical presentation of the dermal occupational exposure limit (DOEL) relative to the dermal dose/unit area $\left(D_{A}\right)$ and the exposed skin surface area $(A)$. The AUC (shaded area) represents the "safe" values for $D_{A} \times A$. For $D_{A}$ $\geqslant b$ (when a maximal flux relevant for the occupational situation $\left(\mathcal{F}_{\text {max;oc }}\right)$ is reached), the DOEL can be set as $A_{\text {max }}$
$(=a)$, and is independent of the dermal dose/unit area. As long as $A<a$, the absorbed dose is not expected to give rise to adverse systemic health effects. If $A>a$, either the exposure time or $D_{A}$ should be reduced. Then, the DOEL can be expressed as the multiplication of the dermal dose/unit area and the exposed skin surface area: $D_{A} \times A$.

be defined (fig 2 in which the theoretical DOEL expressed as $D_{A} \times A$ is graphically presented), so that if $A \leqslant A_{\max }$, no adverse health effects are to be expected because the HBR$\mathrm{OEL}_{\text {int }}$ will then not be exceeded. If $D_{A} \geqslant b$ (the dermal area dose at which $\mathcal{f}_{\text {max; } ; 0 c}$ is reached) a maximal penetration rate is reached, a further increase of $D_{A}$ will not lead to a higher absorbed dose during a specified $T$. The amount taken up is then independent of $D_{A}$ and depends only on the exposed surface area $A$. Thus as long as $A<a$ (fig 2), the absorbed dose will not exceed the HBR-OEL int $_{\text {; }}$ the DOEL can then simply be set as $A_{\max }(=a$, calculated as HBR-OEL int $\left._{\text {in }} /\left(\mathscr{F}_{\text {max; oc }} \times T\right)\right)$. In case $A>A_{\text {max }}$, the internal exposure can be reduced by either diminishing the exposure time $T$ or reducing the flux $\mathcal{F}$. Then, as stated above, $\mathcal{F}$ is dependent on $D_{A}$ and the flux can be reduced by decreasing $D_{A}$. This situation is similar to that described later with an absorption percentage as the starting point, the external DOEL can then be interpreted as the product $D_{A} \times A$ (see later). As long as the value for $D_{A} \times A$ assessed for an occupational situation lies in the shaded area of the curve (the AUC in fig 2), the HBR$\mathrm{OEL}_{\text {int }}$ will not be exceeded.

\section{ASSESSMENT OF A DOEL BASED ON ABSORPTION} PERCENTAGE

If no adequate data are available to estimate an appropriate absorption rate a DOEL has to be based on absorption percentage. As already mentioned, an absorption percentage estimated for a specific $D_{A}$ is not commonly applicable for other values of $D_{A}$; the absorption percentage may increase with decreasing $D_{A}$. The percentage absorbed depends on the

Table 2 Summary of the possibilities for the setting of a dermal occupational exposure limit (DOEL)

\begin{tabular}{ll}
\hline Starting point & DOEL expressed as \\
\hline Biological monitoring & Biological limit value \\
Maximal penetration rate & $A_{\max }$ (if $\mathcal{F}_{\text {max; } ; 0 c}$ is known and applicable) \\
Absorption percentage & $D_{A} \times A$
\end{tabular}

$A=$ exposed surface area; $A_{\max }=$ maximal surface area to be exposed; $\mathcal{F}_{\max ; \text { occ }}=$ maximal flux derived under exposure conditions relevant for the occupational situation; $D_{A}=$ dermal area dose. experimental conditions, including the exposure period and the concentration..$^{15}{ }^{18}$ Without further knowledge, the default value for maximal dermal absorption is set at $100 \%$, unless experimental data or physicochemical parameters may point to a lower maximal absorption percentage (see next section). The $\mathrm{HBR}-\mathrm{OEL}_{\text {int }}$ (mg/day) may then be translated into an external DOEL by dividing the $\mathrm{HBR}_{-} \mathrm{OEL}_{\text {int }}$ through the absorbed fraction $(F)$ of the substance: $\mathrm{HBR}_{-} \mathrm{OEL}_{\text {int }} / F$. This external DOEL can then be interpreted as the product of two variables, the dermal dose/unit area and the exposed surface area $\left(A ; \mathrm{cm}^{2}\right)$ for a given work shift-that is, $D_{A} \times A$.

For testing whether exposure conditions comply with the external DOEL two approaches are now possible. Firstly, a single default value for $A$ can be set for every occupational situation and $D_{A}$ can then be determined at the workplace, or secondly, both parameters may be determined at the actual workplace and the product can be calculated. In the first situation, $D_{A}$ is the single variable to be monitored for compliance. This is a rather conservative and rigid approach. In the second situation both variables are to be monitored and actual exposure conditions can be taken into account.

A default value for $A$ may be based on knowledge of the actual occupational exposure situation. Estimates for the surface area for different parts of the body have been presented. ${ }^{15}$

For a given value of the DOEL (defined as $D_{A} \times A$ (mg/day)) $D_{A}$ is allowed to be higher for smaller values of $A$. As already noted, if $D_{A}$ increases, the absorption percentage will stay equal or will decrease when $\mathcal{F}_{\text {max;occ }}$ is reached. So, if for a given DOEL the actual $A$ is much smaller than the default value on which the DOEL is based the amount of substance absorbed will be overestimated, the overestimation will increase as the actual $A$ deviates more from the default value.

\section{SUMMARY}

The above considerations have been summarised in table 2 . If $\mathcal{F}_{\text {max;occ }}$ can be estimated, a maximal skin surface area to be exposed can be calculated and the DOEL can be expressed as $A_{\max }\left(\mathrm{cm}^{2}\right)$. As long as $A \leqslant A_{\max }$, no adverse health effects are to be expected. If $\mathrm{A}>A_{\max }, D_{A}$ $\left(\mathrm{mg} / \mathrm{cm}^{2}\right)$ has to be estimated and the DOEL can be expressed as $D_{A} \times A(\mathrm{mg})$.

If $\mathcal{f}_{\text {max; } ; \text { cc }}$ is unknown, the skin uptake has to be estimated with a relevant absorption percentage. The DOEL can then be interpreted as $D_{A} \times A$. It is recommended that a DOEL should be derived relative to a standard exposed surface area. If the actual exposed skin surface area deviates from this standard area, the $D_{A}$ can be adjusted accordingly. For instance, for a given maximal value for $D_{A} \times A$ (the DOEL) $D_{A}$ is allowed to be higher if $A$ decreases. But then the absorption percentage may decrease, which means that if the appropriate data are available, a relatively high DOEL may be set for occupational settings where the actual exposed skin surface area is expected to be small, and a relatively low DOEL for a worst case default value for $A$. 
The estimation of dermal uptake with an absorption percentage will be more applicable with a finite dermal dose/unit area, whereas the approach with an absorption rate (flux) can be applied in situations where an infinite dermal dose/unit area is present. ${ }^{18}$ To illustrate this DOELs are calculated for MDA and cyclophosphamide in a later section.

\section{Estimation of dermal penetration}

OCCUPATIONAL FACTORS AFFECTING DERMAL PENETRATION

In the procedure for the assessment of a DOEL as proposed in the previous section, the estimation of dermal uptake, either expressed as a flux or as an absorption percentage, is of crucial importance. Comprehensive reviews on dermal penetration of chemical substances already exist. ${ }^{14-15} 1824$ 29-31 These reviews teach that factors affecting dermal penetration can be divided into three distinct categories: (a) substance related factors - for example, physicochemical properties such as molecular weight, octanol-water partition coefficient, volatility, and polarity (ionisability); (b) situation related factor - for example, environmental temperature, humidity, the presence or absence of occlusive material (clothing, gloves), the time frame of the exposure (duration and frequency)); (c) skin related factors-for example, anatomical site, physically damaged skin.

The compound will in practice be irregularly divided over the exposed skin area. However, the DOEL will usually relate to a continuous 8 hour shift exposure with a constant exposure level. Generally, it can be assumed that when the amount of substance is irregularly divided over the exposed skin surface area, the amount taken up will be equal or less than when the division is regular (under the assumption that the flux is roughly equal over the entire exposed surface area). As a worst case assumption, it is assumed that the total amount is present on the skin during the entire workshift.

In assessing a DOEL it is assumed that dermal penetration is measured for the pure substance. If a substance is part of a mixture, dermal penetration may vary greatly with the actual composition. Also, skin contact may affect the solubility of the compound in the vehicle, and, therefore, the absorption. ${ }^{32}$ It is important to note that not only the dermal dose/unit area, but the concentration in the vehicle at the skin surface is of importance. ${ }^{15}$ For equal absolute amounts of a substance applied per $\mathrm{cm}^{2}$, different concentrations in any vehicle at the skin surface may lead to different absorption rates or percentages. This may at least partly explain the difference in absorption percentages for MDA found by two groups of investigators. ${ }^{33}{ }^{34}$ In the present report, it is assumed that the concentration in the vehicle (solution) is more or less constant. The starting point is a pure substance or a constant concentration of the substance at the skin surface. Furthermore, it should be realised that occupational exposure may be due to contact with contaminated surfaces, gases, aerosols, liquids, and dusts, which will require different approaches in estimating both the actual dermal exposure and its uptake.

Basically, every departure of the skin condition from normal will alter the dermal penetration. ${ }^{18}$ Also, dermal penetration varies with anatomical site. Therefore, by choice, the DOEL relates to normal, healthy adult skin of the volar side of the arm. It is generally not necessary to differentiate DOELs according to race or sex. ${ }^{18}$ However, in vivo experiments indicated increasing lag times (the time before the onset of vasodilatation induced by application of methyl nicotinate) in skin from Asian, white, and black people, respectively, with the lag time in black people being twice as long as in Asian people. ${ }^{35}$

\section{ESTIMATION OF DERMAL PENETRATION}

The estimation of dermal penetration is difficult due to the fact that absorption is influenced by several substance, situation, and skin dependent variables. Recently, US EPA and ECETOC have discussed several in vitro and in vivo techniques for the estimation of skin penetration in detail. ${ }^{15}{ }^{18}$ The reliability of the estimated DOEL will increase if skin uptake can be estimated more precisely for the occupational exposure conditions.

The US EPA proposed several equations for the estimation of the dermally absorbed dose/day for aqueous solutions and vapours. ${ }^{15}$ The US EPA based the estimation of dermal penetration predominantly on the estimation of the permeability constant; equations for the estimation of this constant were presented for aqueous solutions and for vapours for steady state and non-steady state situations. The theoretical and experimental considerations for the estimation of an appropriate permeability constant in different exposure situations were reviewed in detail. The US EPA considered non-steady state conditions to characterise actual exposure more closely than do steady state conditions, especially for exposure periods which are relatively short compared with the lag time necessary to reach a steady state flux..$^{15}$ It is recommended that the usefulness and applicability of these approaches for the occupational situation be studied.

Leung and Paustenbach reviewed some important principles involved in the assessment of percutaneous absorption and discussed some possibilities for a quantitative determination of chemical uptake through the skin. ${ }^{36}$ Although a mathematical model may provide a proper tool for estimating percutaneous absorption, the available models still need further validation (see previous section). The same authors proposed three indirect methods for the estimation of the dermal bioavailability of a substance: by comparison of the area under the plasma concentration-time curve after cutaneous and intravenous administration (preferably performed with labelled material), by estimating the total amount excreted relative to the administered dose, and by measurement of the amount of substance remaining on the skin at the end of exposure. ${ }^{31}$ The first method may be the most accurate; 
however, these data are seldom available in risk assessment.

Recently, a tiered approach for the estimation of dermal absorption has been described. ${ }^{19}$ Briefly, this approach starts with a default value of $100 \%$ for dermal absorption when no data are available. In the next tier molecular weight or the $\log P_{o w}$ were proposed to discriminate between poorly absorbed substances, and substances for which $100 \%$ absorption may be a reasonable estimate. It was stated that although no clear relation was presented, absorption would be considerably $<100 \%$ if the molecular weight is greater than 500. For $\log P_{o w}$, maximum absorption was associated with values between 1 and 2 , whereas for $\log P_{o w}$ values $<-1$ or $>4$ the dermal absorption was considered to be $<10 \%{ }^{18}{ }^{19}$

Formulas for the calculation of the flux or the permeation coefficient based on the $\log P_{o w}$ and the molecular weight have been proposed. ${ }^{13}$ However, the US EPA warns that the $\log P_{o w}$ will not be a valid parameter for the estimation of lipophilicity for certain classes of chemicals-for example, nitrophenols. ${ }^{15}$

Recently, the validity of five of these models has been evaluated by comparison with experimental in vitro permeation coefficients by Wilschut et al. ${ }^{37}$ They considered a revised version of an unpublished model by Robinson to be the most appropriate for the estimation of skin penetration from aqueous solutions. However, the usefulness of this model in actual occupational situations remains to be investigated.

The usefulness of in vitro and in vivo test systems has been reviewed recently. ${ }^{15} 18$ The results of in vitro studies for the determination of skin penetration are difficult to compare with those obtained in vivo. Standardised experimental conditions and the use of reference compounds for calibration should improve the comparability of the test systems. In general, animal skin seems to be more permeable than human skin. ${ }^{15}{ }^{18} \mathrm{~A}$ single default correction factor cannot be derived, because the extent of overestimation seems to be specific to the agent and animal. We recommended that animal absorption data is considered as an overestimation of absorption in humans. ${ }^{15} 1819$

Testing of the applicability of the concept The applicability of the procedure described for setting a DOEL was tested by assessing a DOEL for two genotoxic carcinogens cyclophosphamide and MDA. The principle is similar for compounds for which a threshold value for the expression of toxic effects can be assessed. The assessments based on literature searches are briefly described, more details are reported separately (in Dutch). ${ }^{21}{ }^{38}$ For both compounds, relevant dermal toxicity data were


ing point. These values were calculated according to the Dutch method for the calculation of health based calculated occupational cancer risk values (HBC-OCRV) for genotoxic carcinogens, ${ }^{39}$ as adopted by DECOS, a commission of the Health Council of the Netherlands.
DERIVATION OF A DOEL FOR CYCLOPHOSPHAMIDE Risk evaluation

Key study for the risk evaluation of cyclophosphamide was the oral experiment with rats by Schmähl and Habs. ${ }^{40}$ Under the assumption of $100 \%$ oral absorption and based on the previously mentioned method for derivation of an HBC-OCRV for genotoxic carcinogens ${ }^{41}$ an incidence of $0.97 / \mathrm{mg}$ cyclophosphamide absorbed $/ \mathrm{kg} /$ day for malignant tumours was calculated for male rats. The incidence per $\mathrm{mg}$ absorbed cyclophosphamide per day for the worker $(70 \mathrm{~kg} ; 40$ year exposure for five days/week) is then $5.3 \times 10^{-3}$. Reference values for a working life (40 years) additional mortality incidences of 4/1000 and 4/100 000 are requested by the Dutch Ministry of Social Affairs and Employment. The daily internal cyclophosphamide doses associated with these excess cancer levels are $0.75 \mathrm{mg}$ and $7.5 \mu \mathrm{g}$, respectively.

\section{Dermal absorption}

Key studies for the estimation of dermal absorption were the volunteer studies of Hirst et $a l^{41}$ the studies with cancer patients by Mouridsen et $a l,{ }^{42}{ }^{43}$ and the study with rats by Sessink et al. ${ }^{44}$ The animal study showed a urinary cyclophosphamide excretion of $5 \%-7 \%$ after dermal and intravenous administration, indicating $100 \%$ dermal absorption. The human studies indicated that after intravenous and dermal administration, about $10 \%$ and $1 \%$ of the administered dose, respectively, was excreted in the urine in 24 hours as cyclophosphamide. The human and rat data are difficult to compare, due to significant differences in exposure conditions. In the rat study cyclophosphamide was applied in a glycerol suspension which penetrates easily into the skin, and therefore may have enhanced the absorption. In the human volunteer study, cyclophosphamide was applied in methanol which evaporated within 30 seconds, the skin was covered and washed with water and soap after 6 hours. As the exposure conditions in the human volunteer study were considered to be more comparable with the occupational conditions (watery solutions; exposure for 6 hours before cleaning) this study was used as a starting point for the estimation of the absorption.

Based on these human studies the total urinary excretion of cyclophosphamide within 4 days after intravenous administration (0.02 $\mathrm{mg} / \mathrm{kg}$ ) was about $13 \%$. A total of $2 \%-3 \%$ cyclophosphamide was estimated to be excreted in urine after dermal application of $1 \mathrm{mg}$ $\left(100 \mu \mathrm{g} / \mathrm{cm}^{2}\right.$; occlusion). So, urinary cyclophosphamide excretion after dermal application was maximally $25 \%-30 \%$ of that after a comparable intravenous dose.

Estimation of a DOEL for actual exposure For preparation and application of cyclophosphamide as a drug it is expected that dermal exposure is limited to the hands and lower arms, an area of about $2000 \mathrm{~cm}^{2}$. Starting from an absorption percentage of $30 \%$, a daily internal dose of $0.75 \mathrm{mg}$ equals a $D_{A}$ of $(750 \times 100 /$ $30) / 2000 \approx 1 \mu \mathrm{g} / \mathrm{cm}^{2}$. However, the absorption 
percentage of $30 \%$ was estimated based on a $D_{A}$ of $100 \mu \mathrm{g} / \mathrm{cm}^{2}$. Considering the fact that absorption percentage may increase with decreasing $D_{A}$, an absorption percentage of $100 \%$ is assumed at a dermal dose/unit area of about $1 \mu \mathrm{g} / \mathrm{cm}^{2}$. Therefore, the DOEL interpreted as $D_{A} \times A$, was set at $0.75 \mathrm{mg} /$ day. For an estimated maximum value for $A$ of $2000 \mathrm{~cm}^{2}$ the $D_{A}$ will be $750 / 2000 \approx 0.4 \mu \mathrm{g} / \mathrm{cm}^{2}$. Similarly, the cyclophosphamide dose of $7.5 \mu \mathrm{g}$ associated with the lower reference value equals a $D_{A}$ of $4 \mathrm{ng} / \mathrm{cm}^{2}$.

DERIVATION OF A DOEL FOR 4,4'-METHYLENE DIANILINE

Risk evaluation

The oral NTP study was the main source of data for the evaluation of the carcinogenic risk of MDA. ${ }^{45}$ Under the assumption of $100 \%$ oral absorption and based on the method for derivation of a HBC-OCRV for genotoxic carcinogens already mentioned ${ }^{39}$ an incidence was found of $4.67 \times 10^{-2}$ per mg MDA absorbed $/ \mathrm{kg} /$ day for neoplastic noduli for male rats. The incidence per mg absorbed MDA per day for a worker (70 kg; 40 year exposure for five days/week) is then $0.25 \times 10^{-3}$. The daily internal MDA doses associated with the reference values of 4/1000 and 4/100 000 are $16 \mathrm{mg}$ and $0.16 \mathrm{mg}$, respectively.

\section{Dermal absorption}

The penetration of MDA in the skin is fast but not complete, even after application of low doses about $50 \%$ could be washed off. Generally, the absorption percentage decreased with increasing doses although the absolute amount taken up remained more or less the same. In vitro studies showed that about twice as much MDA penetrates human skin as rat skin. Occlusion increased the amounts taken up by twofold to 2.5 -fold. ${ }^{46}$

Absorption studies with volunteers have been carried out. Exposure conditions in most studies deviate from occupational conditions. In one experiment with exposure conditions resembling those at the workplace MDA $(10 \%$ solution $(\mathrm{w} / \mathrm{v})$ in ethanol; $\left.D_{A} \approx 0.6 \mathrm{mg} / \mathrm{cm}^{2}\right)$ was applied to the forearm of two volunteers, without occlusion. After three hours $41 \%$ and $47 \%$, respectively, could be washed off. ${ }^{47}$ Based on these results an absorption percentage of $55 \%$ was derived for MDA.

\section{Estimation of a DOEL for actual exposure}

In general, occupational skin exposure is limited to hands and lower arms, an area of $2000 \mathrm{~cm}^{2}$. Starting from an absorption percentage of $55 \%$, a daily internal dose of $16 \mathrm{mg}$ equals a $D_{A}$ of $(16000 \times 100 / 55) / 2,000 \approx 15 \mu \mathrm{g} / \mathrm{cm}^{2}$. However, the absorption percentage of $55 \%$ was estimated based on a $D_{A}$ of $0.6 \mathrm{mg} / \mathrm{cm}^{2}$. Considering the fact that the absorption percentage for MDA will increase with decreasing dermal dose/unit area, an absorption percentage of $100 \%$ is assumed at a dermal dose/unit area of about 15 $\mu \mathrm{g} / \mathrm{cm}^{2}$. Therefore, the DOEL interpreted as $D_{A} \times A$ was set at $16 \mathrm{mg}$ a day. For an estimated maximum value for $A$ of $2000 \mathrm{~cm}^{2}$ the $D_{A}$ will be $16000 / 2000 \approx 8 \mu \mathrm{g} / \mathrm{cm}^{2}$. Similarly, the MDA dose of $0.16 \mathrm{mg}$ associated with the lower refer- ence value equals a dermal dose/unit area of 80 $\mathrm{ng} / \mathrm{cm}^{2}$.

\section{Discussion and conclusions}

The present report presents a first attempt to develop a procedure for the assessment of a relevant and useful quantitative parameter for controlling dermal exposure. It is meant as a starting point for further discussion to develop a practically suitable and applicable DOEL for occupational situations. The importance of the contribution of skin uptake to the total human exposure for many chemicals is widely recognised. ${ }^{1011} 1518$ Initiatives in the United States ${ }^{10}$ and the establishment of a European network on dermal exposure supported by the European Commission should provide important information in the near future for refinement of the proposed procedure. For some chemicals in the workplace and for certain workplaces skin uptake will be the predominant or only exposure route.

Dermal exposure is merely controlled by a qualitative parameter. One of the reasons for this is that the problems associated with quantitative standard setting for dermal exposure are thought to be complex. It is acknowledged that for the proposed procedure several assumptions and simplifications had to be made. However, many of the drawbacks and assumptions mentioned are not unique to dermal exposure and are basically not different from those associated with oral or respiratory exposure. For instance, in setting standards for occupational respiratory exposure, combined exposure is seldom dealt with, ventilation rate (and, therefore, uptake) may be concentration dependent, ${ }^{48}$ work load and working conditions may influence breathing rate and depth, and the ratio of mouth versus nose breathing may also be of importance. Furthermore, pharmacokinetic data for respiratory exposure are often lacking and default values for absorption have to be used. All these factors, which are seldom accounted for, will determine the pulmonary uptake of chemical substances, and therefore the internal dose.

Also, in respiratory exposure the amount actually absorbed is for some substances related to the proportions of differently sized particles, defining the inhalable and respirable fraction. Certain groups of substances-for example, fibres, dusts, and aerosols-require a specific approach. It may, therefore, be relevant for health risk assessment to consider whether a substance is taken up by the nasal mucosa or the alveoli.

Thus, also for other routes of exposure, absorption will be dependent on characteristics related to substances and situations and to physiological variables. Nevertheless, many OELs for respiratory exposure serve as useful tools for regulating exposure to prevent adverse health effects in workers.

In table 2, the possible assessments of a quantitative DOEL are summarised. The total internal exposure (including dermal) may be controlled by a BLV. The suitability of a BLV for this purpose will be dependent on the usefulness of specific variables in biological tissues 
and the availability of an appropriate method for analysis. Often, a specific strategy for sampling biological fluids and analysis will be demanded, especially when both respiratory and dermal exposure are of importance. Both routes will probably result in different internal exposure patterns and, therefore, different urinary excretion patterns.

If an external DOEL is considered to be appropriate we recommend assessment of this DOEL relative to the surface area of exposed skin. Generally, the DOEL will be derived from an HBR-OEL $_{\text {int }}$ derived from animal toxicity data. Translation to an external DOEL is preferably performed with an appropriate estimation of the penetration rate of the substance under occupational conditions. If a $\mathcal{F}_{\text {max; } ; \text { cc }}$ can be estimated it may be possible to define a maximum skin surface area $\left(A_{\max }\left(\mathrm{cm}^{2}\right)\right)$ to be exposed for a given $T$. If the actual exposed surface area is $\leqslant A_{\max }$ no health risk is indicated for dermal exposure, independent of the dermal dose/unit area. If a $\mathcal{F}_{\text {max;occ }}$ cannot be derived or if $A>A_{\max }$ the approach will be the same as when an absorption percentage serves as a basis, the DOEL can then be interpreted as $D_{A} \times A$. Compliance can then be tested by monitoring either both $D_{A}$ and $A$ or only $D_{A}$ starting from a default value for $A$. If for a given DOEL $A$ decreases, $D_{A}$ is allowed to increase. Hence, if the actual exposed surface area-for example, hand only exposure-is much smaller and the $D_{A}$ is allowed to be, and the $D_{A}$ is much higher than the initial values on which the DOEL is based, lower absorption percentages can be used in assessment of health risk. This means that for a relatively small area of exposed skin the DOEL expressed as $D_{A} \times A$, may be set at a higher level. So, for a specific substance the DOEL may be set at different levels depending on the actual surface area of exposed skin.

A quantitative DOEL can be used to control dermal exposure, but also, in combination with dermal exposure data to rank substances for possible risks at the workplace. Also, it might be helpful to manufacturers of personal protective equipment for the development of appropriate products.

The present concept is mainly developed for substances that act systemically. In general, the occurrence of local effects will be predominantly dependent on $D_{A}$, the actual concentration at the skin surface. Therefore, if a dose-effect relation for local effects is available, local effects may be regulated by assessing a maximum value for $D_{A}$ as well as a maximal value for the DOEL expressed as $D_{A} \times A$.

A quantitative DOEL will be a useful type of exposure limit complementary to respiratory OELs at the workplace. Together they may provide a more complete set of exposure limits which allow assessment of a worker's health risk relative to chemical exposure at work. This is at least the case for those occupational situations where the uptake through respiratory exposure is far less than through dermal exposure. An integrated approach, summation of the internal exposures through both routes, is recommended for situations where both exposure routes contribute considerably to the internal exposure of the worker. In that case, a DOEL as well as a respiratory OEL may be set, taking into account that combined exposure through the dermal and respiratory route at the same time should not result in a health risk. Exposure to both routes should be controlled together. A concept for a procedure for this should be developed taking the route specific kinetics into account. It will, however, be difficult to assess whether the critical effect is related to dose or concentration. If related to dose, the absorbed doses may be summed, whereas, if related to concentration it should be realised that the internal exposure pattern (height, onset, and duration of measured concentrations of blood and tissue) may be completely different for both routes. In our institute this is currently under study.

A DOEL derived according to the proposed concept may already be useful as is illustrated by the evaluation of an external DOEL for MDA in the workplace investigated. ${ }^{12}$ In this study a significant correlation was found between cumulative MDA excretion in urine and the results of the dermal exposure measurements (assessed by hand washing). Testing for compliance was performed both by comparison between the MDA excreted in the urine and a BLV, and by comparison between the estimated external dermal exposure (hand wash method) and the external DOEL as derived in this report. Both comparisons were in close agreement, the exposure was estimated to be $20 \%-25 \%$ of the BLV and DOEL, respectively. ${ }^{12}$

As stated before, one of the first steps now is to develop criteria to set a priority list of chemicals for which a DOEL is a useful and necessary tool in occupational health risk management. For this purpose the publications of ECETOC and US EPA and others may provide some basic elements, but these need further development and evaluation. ${ }^{9} 1531$

Of crucial importance for a proper assessment of a DOEL for systemic effects is the estimation of dermal uptake. If no relevant data on dermal absorption are available a default value of $100 \%$ may be used. If relevant physicochemical data are available a maximal expected absorption of $<100 \%$ may be calculated. Some proposals have been made for the estimation of dermal penetration. ${ }^{15} 18193136$ Further, recent comparison of a few models based on molecular weight and $\log P_{\text {ow }}$ led to a proposal for an additional model for the estimation of skin penetration for aqueous solutions. ${ }^{37}$ A combined evaluation of the applicability of the proposed models and procedures for general and specific occupational environments may provide some useful tools for a protocol for the estimation of dermal penetration at the workplace and, therefore, of a more refined DOEL.

In summary, although several assumptions and simplifications have been made, the proposed concept is considered workable in an occupational environment. The extent and complexity of the problems associated with the assessment of a DOEL do not mean that applicable and relevant standards cannot be derived 
for skin exposure. Also during standard setting for respiratory and oral exposure, interpretational and applicability problems often have to be dealt with. Tentative DOELs were derived for two carcinogens, the procedure will be similar for non-neoplastic agents. Furthermore, the DOEL seemed to be relevant and applicable for MDA in an actual occupational setting. ${ }^{12}$ We recommend the testing of this approach for more substances in practice.

This study was financially supported by the Dutch Ministry of Social Affairs and Employment. The important contributions of NJ van Sittert (Shell International Chemicals BV, Department of Molecular Toxicology, Amsterdam) and $\mathrm{H}$ van der Waal (Shell Nederland Chemie BV, Biomedical Laboratory, Pernis) in parts of this work are gratefully acknowledged, as well as the valuable contributions of JJG Opdam, J Krüse, and WK de Raat (TNO Nutrition and Food Research Institute, Zeist). One of the referees of the present manuscript is gratefully acknowledged for his valuable comments.

1 American Conference of Governmental Industrial Hygienists (ACGIH). Documentation of the threshold limit values and biological exposure indices, 6th ed. Cincinnati, USA: ACGIH, biological 1991 .

2 Greim H, Lehnert G, eds. Biologische Arbeitsstoff-ToleranzWerte (BAT-Werte) und Expositionsäquivalente für krebserzeugende Arbeitsstoffe (EKA). Weinheim, Germany: VCH Verlagsgesellschaft mbH, 1996.

3 Wilson HK. Recent policy and technical developments in biological monitoring in the United Kingdom. Sci Total Environ 1997;199:191-6.

4 Aitio A, Pekari K, Järvisalo J. Skin absorption as a source of error in biological monitoring. Scand $\mathcal{F}$ Work Environ Health 1984;10:317-20

5 Kao J, Carver MP. Cutaneous metabolism of xenobiotics. Drug Metab Rev 1990;22:363-410.

6 Paustenbach DJ. Assessment of the developmental risks resulting from occupational exposure to select glycol ethers within the semiconductor industry. F Toxicol Environ Health 1988;23:29-75.

7 Grandjean P, Berlin A, Gilbert M, et al. Preventing percutaneous absorption of industrial chemicals: the skin denotaneous absorption of industrial chem

8 Scansetti G, Piolatto G, Rubino G. Skin notation in the context of workplace exposure standards. Am f Ind Med 1988; 14:725-32.

9 European Centre for Ecotoxicology and Toxicology of Chemicals. Strategy for assigning a skin notation. Brussels: ECETOC, 1993 (Revised ECETOC Document No 31.)

10 Fenske R, Van Hemmen, JJ. Occupational skin exposure to chemical substances: setting limits [editorial]. Ann Occup Hyg 1994;38:333-6.

11 Dost AA. Meeting report. A European meeting held to discuss dermal exposure monitoring and related issues. Brussels: 21-23 June 1994. Ann Occup Hyg 1995;39:24155.

12 Brouwer D, Hoogendoorn L, Bos PMJ, et al. Proposal for the assessment of quantitative dermal exposure limits in the occupational environment. Part II. Feasibility study for application in an exposure scenario for MDA by two differapplication in an exposure scenario for MDA by two differMed 1997;55:000-000.

13 Fiserova-Bergerova V, Pierce JT, Droz PO. Dermal absorption potential of industrial chemicals: criteria for skin notation. Am F Ind Med 1990;17:617-35.

14 Fiserova-Bergerova V. Relevance of occupational skin exposure. Ann Occup Hyg 1993;37:673-85.

15 Environmental Protection Agency. Dermal exposure assessment: Principles and applications [interim report]. Washington, DC: US Environmental Protection Agency, 1992.

16 Kežić S, Mahieu K, Monster AC, et al. Dermal absorption of vaporous and liquid 2-methoxyethanol and $38-43$.

17 Johanson G, Boman A. Percutaneous absorption of 2-butoxyethanol vapour in human subjects. $\mathrm{Br} \mathcal{F}$ Ind $\mathrm{Med}$ 1991;48:788-92.

18 European Centre for Ecotoxicology and Toxicology of Chemicals. Percutaneous absorption. Brussels: ECETOC, 1993:1-80. (Monograph No 20.)

19 Stevenson H, Opdam JJG, Van Ommen B, et al. Protocol for the estimation of dermal absorption according to a tiered approach on behalf of the risk assessment of pesticides. Zeist, The Netherlands: TNO, 1994. (TNO-report V94.129.)

20 Bronaugh RL, Barton CN. Prediction of human percutaneous absorption with physicochemical data. In: Wang RGM Knaak JB, Maibach HI, eds. Health risk assessment. Dermal and inhalation exposure and absorption of toxicants. Boca Raton, USA: CRC Press, 1993.

21 Bos PMJ. Testing the possibility for the assessment of a dermal occupational exposure limit: a case study. Cyclophosphamide. Zeist, The Netherlands: TNO Food and Nutrition Research, 1994. (In Dutch.)
22 Fenske R. Dermal exposure assessment techniques. Ann Occup Hyg 1993;37:687-706.

23 Cherrie, JW, Robertson A. Biologically relevant assessment of dermal exposure. Ann Occup Hyg 1995;39:387-92.

24 Hotchkiss SAM. Skin absorption of occupational chemicals. In: Handbook of occupational hygiene. Kingston upon Thames, UK: Groner, 1993.

25 Chester G. Revised guidance document for the conduct of field studies of exposure to pesticides in use. In: Curry PB, Iyengar S, Maloney P, Maroni M, eds. Methods of pesticide exposure assessment. London: Plenum Press, 1995.

26 Van Hemmen JJ, Van Golstein Brouwers YGC, Brouwer $\mathrm{DH}$. Pesticide exposure and re-entry in agriculture. In: Curry PB, Iyengar S, Maloney PA, Maroni M, eds. Methods of pesticide exposure assessment. London: Plenum Press, 1995.

27 De Raat WK, Krüse J, Stevenson H, et al. Outline of a protocol for dermal-to-dermal extrapolation. TNO-report V97.972, Zeist, The Netherlands: 1997. (TNO-report V97.972.)

28 Hakkert BC, Stevenson H, Bos PMJ, et al. Methods for the establishment of health-based recommended occupational exposure limits for existing substances. Zeist, The Netherlands: 1996. (TNO-report V96.463.)

29 Wester RC, Maibach HI, Cutaneous pharmacokinetics: 10 steps to percutaneous absorption. Drug Metab Rev 1983;14:169-205.

30 Grandjean P. Skin penetration: Hazardous chemicals at work. London: Taylor and Francis, 1990.

31 Paustenbach DJ, Leung H-W. Techniques for assessing the health risks of dermal contact with chemicals in the environment. In: Wang RGM, Knaak JB, Maibach HI, eds. Health risk assessment. Dermal and inhalation exposure and absorption of toxicants. Boca Raton, USA: CRC Press, 1993.

32 De Carvalho M, Falson-Rieg F, Eynard I, et al. Changes in vehicle composition during skin permeation studies. In: Brain KR, James VJ, Walters KA, eds. Prediction of percutaneous penetration. Vol 3b. Cardiff, UK: STS Publishing, 1993:251-54.

33 Boogaard PJ, Van der Waal H. Biological monitoring of dermal exposure to 4,4'-diaminodiphenyl methane $(M D A)$ by determination of MDA in hydrolysed urine- a human volunteer study. Shell Biomedical Laboratory, preliminary internal report. Rotterdam, The Netherlands: Shell Biomedical Laboratory, 1994.

34 Brunmark P. Methods for assessment of exposure to aromatic amines and isocyanates by air monitoring and biomarkers [doctoral dissertation]. Lund, Sweden: University of Lund, 1994.

35 Kompaore F, Tsuruta H. In vivo differences between Asian, black, and white in the stratum corneum barrier function. Int Arch Occup Environ Health 1993;65:S223-5.

36 Leung H-W, Paustenbach DJ. Techniques for estimating the percutaneous absorption of chemicals due to occupational and environmental exposure. Appl Occup Environ Hyg 1994;9:187-97.

37 Wilschut A, Ten Berge WF, Robinson PJ, et al. Estimating skin permeation. The validation of five mathematical skin permeation models. Chemosphere 1995;30:1275-96.

38 Boogaard PJ. Testing the possibility for the assessment of a dermal occupational exposure limit: a case study. 4,4'-methylene dianiline. Den Haag, The Netherlands: Shell Internationale Petroleum Maatschappij B, 1994. (Unpublished report, in Dutch.)

39 Health Council of the Netherlands: Dutch Expert Committee on Occupational Standards (DECOS): Calculating cancer risk. The Hague: Health Council of the Netherlands, 1995. (Publication No 1995/06WGD.)

40 Schmähl D, Habs M. Carcinogenic action of low-dose cyclophosphamide given orally to Sprague-Dawley rats in a lifetime experiment. Int $\mathcal{F}$ Cancer 1979;23:706-12.

41 Hirst M, Tse S, Mills DG, et al. Occupational exposure to cyclophosphamide. Lancet 1984;i:186-8.

42 Mouridsen HT, Faber O, Skovsted L. The biotransformation of cyclophosphamide in man: analysis of the variation in normal subjects. Acta Pharmacol 1974;35:98-106.

43 Mouridsen HT, Faber O, Skovsted L. The metabolism of cyclophosphamide. Dose dependency and the effect of long-term treatment with cyclophosphamide. Cancer 1976; 37:665-70.

44 Sessink PJM, Van den Broek PHH, Bos RP. Urinary cyclophosphamide excretion in rats after intra tracheal, dermal, oral, and intravenous administration of cyclophosphamide. f Appl Toxicol 1991;11:125-8.

45 NTP. Technical Report on the carcinogenesis studies of 4,4'-methylene dianiline dihydrochloride in F344/N rats and $\mathrm{B}_{6} \mathrm{C} 3 \mathrm{~F}_{1}$ mice (drinking water studies). NTP, 1983. (Cas No 13552-44-8.)

46 Hotchkiss SAM, Hewitt P, Caldwell J. Percutaneous absorption of 4,4 '-methylene-bis-(2-chloroaniline) and 4,4 '-methylene dianiline through rat and human skin in vitro. Toxicology in Vitro 1993;2:141-8.

47 Boogaard PJ, Van der Waal, H. Biological monitoring of dermal exposure to 4,4'-diamino diphenylmethane $(M D A)$ by determination of $M D A$ in hydrolysed urine- a human volunteer study. Rotterdam, The Netherlands: Shell Biomedical Laboratory, preliminary internal report, 1994.

48 Alarie Y. Sensory irritation by airborne chemicals. Crit Rev Toxicol 1973;2:299-363. 